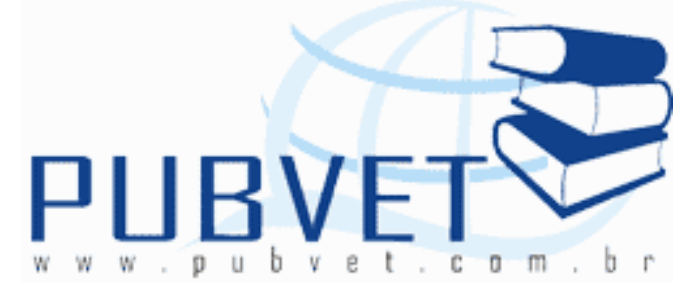

PUBVET, Publicações em Medicina Veterinária e Zootecnia.

\title{
A importância do cálcio e fósforo na nutrição de psitacídeos e passeriformes - uma revisão
}

\author{
Ricardo Baggio Júnior ${ }^{1}$ e Maria Carolina Gonçalves Pita²
}

${ }^{1}$ Discente do 90 semestre de Medicina Veterinária - Universidade Guarulhos 2Docente da Universidade Guarulhos

\section{Resumo}

O cálcio e o fósforo são os maiores constituintes minerais do corpo animal e devem estar balanceados no organismo, pois tanto o excesso quanto a deficiência de um desses dois minerais implica em doenças nutricionais e limita o desempenho das aves. O paratormônio e a calcitonina configuram-se como os principais hormônios reguladores dessa relação Ca:P. O cálcio é importante para a coagulação sanguínea, a excitabilidade de nervos e músculos, o ín mensageiro intracelular, a formação da casca do ovo, a ativação enzimática e a contração muscular. O fósforo, por sua vez, está envolvido em muitas funções do organismo, tais como no metabolismo energético de carboidratos, de gorduras e de aminoácidos, no equilíbrio acidobásico, entre outros. A falta de informação por parte de proprietários e veterinários, a respeito dos requerimentos nutricionais, assim como teores de nutrientes dos alimentos oferecidos às aves, são os principais fatores que levam a distúrbios nutricionais. Em situações práticas, o cálcio, o fósforo e a vitamina $D$ são os nutrientes mais frequentemente envolvidos nas osteodistrofias, sendo estas 
classificadas em osteopenias (osteoporose, raquitismo) ou osteomegalias (condrodistrofia).

Palavras-chave: aves, cálcio, fósforo, vitamina D, dieta.

\title{
The calcium and phosphorus importance in the nutrition of psittaciforms and passerine birds - a review
}

\begin{abstract}
Calcium and phosphorus are the major minerals in the animal body and must be balanced. The excess or deficiency of either results in nutritional diseases and limits the performance of birds. Parathyroid hormone and calcitonin appear as the main regulatory hormones of $\mathrm{Ca}$ : $\mathrm{P}$ ratio. Calcium is important for blood clotting, nerves and muscles excitability, ion as intracellular messenger, eggshell formation, enzyme activation and muscle contraction. Phosphorus, in turn, is involved in many functions of the body, such as in the energetic metabolism of carbohydrates, fats and amino acids, in the acid-base balance, among others. The lack of information of owners and veterinarians about the nutritional requirements and nutrient content of bird feeding are key factors that lead to nutritional disorders. In practical situations, calcium, phosphorus and vitamin $D$ are the nutrients most commonly involved in osteodystrophies, which are classified into osteopenias (osteoporosis, rickets) or osteomegalias (condrodistrophy).
\end{abstract}

Keywords: birds, calcium, phosphorus, vitamin D, diet.

\section{Introdução}

As deficiências nutricionais ocorrem devido a diversos fatores, tais como o desconhecimento das necessidades nutricionais dos animais, de seu comportamento alimentar e da seletividade dos alimentos em cativeiro, além da falta de informações sobre os hábitos alimentares em vida livre de cada espécie. Informações a respeito da composição química e da digestibilidade 
dos alimentos oferecidos são, da mesma forma, de grande importância (Carciofi e Oliveira, 2007).

A alimentação de animais silvestres de diferentes espécies em cativeiro é um desafio, pois tanto as necessidades nutricionais quanto as manifestações clínicas associadas às deficiências variam de espécie para espécie. De acordo com Kollias (1995), psitacídeos apresentam sintomas cutâneos diferentes da galinha doméstica em resposta à deficiência de aminoácidos e vitaminas.

O cálcio e o fósforo são os maiores constituintes minerais do corpo animal. Ao redor de $99 \%$ do cálcio e $80 \%$ do fósforo encontram-se no tecido ósseo, conferindo-Ihe rigidez. O cálcio é também importante para a coagulação sanguínea, a excitabilidade de nervos e músculos, para o ín mensageiro intracelular, a formação da casca do ovo, a ativação enzimática e a contração muscular (Underwood, 1999; Macari et al., 2002). Está presente em três frações no soro das aves: sal ionizado, ligado às proteínas e vinculado a uma variedade de ânions como citrato, bicarbonato e fosfato (Duncan e Prasse, 1986; Sena e Bowers, 1988; Bush, 1991; Nelson et al., 1994). O fósforo está envolvido em quase todos os aspectos do metabolismo animal, incluindo o metabolismo energético (integrante da adenosina trifosfato - ATP), a contração muscular, o funcionamento do tecido nervoso, o metabolismo de carboidratos, as gorduras e aminoácidos, o equilíbrio acidobásico, o transporte de metabólitos, e na estruturação de ácidos nucleicos e da membrana lipoproteica das células (Runho et al., 2001). Nos ossos estes minerais apresentam-se, principalmente, na forma de cristais de hidroxiapatita (Association of American Feed Control Officials Incorporated, 1998).

De acordo com Godoy e Cubas (2006), o excesso tanto de cálcio como de fósforo nutricional leva à formação de fosfato de cálcio insolúvel no sistema digestivo e consequente indisponibilidade do mineral que estiver em menor concentração. Desse modo, tanto a concentração absoluta de cálcio e fósforo como a relação entre eles são importantes. Em uma dieta normal, a relação entre Ca: $P$ recomendada para aves varia de $1: 1,2: 1$ e 3:2, e a concentração de cálcio não precisa ser superior a $1 \%$ na dieta para aves em fase de 
manutenção. Já para Petrack (1982) e Walsh (1985), a relação entre Ca:P deve se aproximar de $1,5: 1$, o que equivale a uma dieta contendo $1 \%$ de cálcio e $0,7 \%$ de fósforo.

O controle do metabolismo do cálcio nas aves é um sistema homeostático altamente eficiente, capaz de responder rapidamente à crescente demanda de cálcio necessário para produção de ovos e ao crescimento quando jovens (Bentley, 1998; Dacke, 2000).

\section{Hormônios envolvidos}

Segundo Carciofi e Oliveira (2007), devido à importante ação do cálcio no funcionamento neuromuscular, faz-se necessário que a concentração plasmática do elemento seja estreitamente regulada. O hormônio da paratireoide (PTH), os metabólitos de vitamina D3 e a calcitonina regulam o cálcio nas aves, assim como ocorre em mamíferos, agindo principalmente sobre o fígado, os rins, órgãos do trato gastrointestinal e ossos (Koch, 1984; Taylor e Dacke, 1984; Bentley, 1998; Dacke, 2000).

Segundo Petrack (1982) e Buckley (1984), a produção de PTH é influenciada por dois principais reguladores: cálcio ionizado e adenosina 3', $5^{\prime}$ monofosfato cíclico (CAMP). À medida que se diminui o Ca ou se aumenta a CAMP, a glândula paratireoide produz mais PTH, estimulando a retirada de cálcio da reserva óssea, bem como o aumento da absorção renal de cálcio e o aumento da excreção de fósforo pelo epitélio tubular renal (McDonald, 1988). A secreção é inibida quando o cálcio em concentração no sangue aumenta ou quando a glândula paratireoide capta uma elevação nas concentrações de calcitriol (1,25 diidroxicolecalciferol, a forma ativa da vitamina D). Dessa forma, o paratormônio atua quando o cálcio da dieta é baixo e existe uma tendência à sua diminuição plasmática (Carciofi e Oliveira, 2007).

As aves parecem ser mais sensíveis ao PTH do que os mamíferos, reagindo às injeções intravenosas do hormônio em questão de minutos, apresentando um aumento imediato do nível de cálcio no sangue (Koch, 1984; Bentley, 1998; Dacke, 2000). Petrack (1982) e Stunkard (1984) também 
sugerem que nas aves o PTH e o estrogênio possuem um efeito sinérgico sobre os níveis de cálcio ionizado.

A glândula Ultimobranquial em aves produz a calcitonina, um hormônio ácido 32-amino que atua diminuindo a atividade dos osteócitos e osteoclastos, reduzindo, com isso, a saída de cálcio dos ossos (Carciofi e Oliveira, 2007), exercendo essencialmente o efeito de hipocalcemia em resposta ao aumento sérico de cálcio ionizado (Dacke, 2000).

As prostaglandinas, por seu turno, são poderosas substâncias endógenas facilitadoras da reabsorção óssea, agem nos osteoclastos e possuem efeitos hipercalcêmicos semelhantes aos do PTH e dos metabólitos da Vitamina D3 (Stanford, 2006). Injeções de prostaglandina em frangos produzem hipercalcemia, e o uso de antagonistas das prostaglandinas levam à hipocalcemia (Dacke, 2000).

\section{Vitamina D}

A vitamina $D$ é um esteroide essencial na regulação da absorção de cálcio pelo trato digestivo e deposição/reabsorção de cálcio nos ossos (Earle e Clarke, 1991). Segundo Aslam et al. (1998), além das funções citadas anteriormente, a vitamina D3 possui profundos efeitos sobre o sistema imunológico, a pele e as células cancerígenas.

De acordo com McDonald (2006) e Leeson e Summers (2001), uma vez que a Vitamina D3 (colecalciferol) é absorvida pelo trato intestinal no duodeno, faciltada pela presença de bile e fomação de micelas ou sintetizada na pele, a mesma é carreada pelos quilomícrons até o fígado onde é hidroxilada e estocada. Para que seja biologicamente ativa, a Vitamina D3 deve sofrer hidroxilação, que ocorre nos rins sob o estímulo do paratormônio.

A vitamina $D$ atua elevando as concentrações plasmáticas de cálcio e fósforo, aumentando a reabsorção renal e a absorção intestinal desses minerais. No intestino, estimula a transcrição e síntese da proteína transportadora de cálcio, sem a qual não existe absorção ativa desse elemento (Leeson e Summers, 2001). Portanto, a deficiência de vitamina D, seja pela 
ingestão insuficiente ou pela produção insuficiente na pele devido à ausência de raios solares ou luz ultravioleta, causa má absorção de cálcio no intestino, impedindo o desenvolvimento e a calcificação normais do tecido ósseo (Godoy e Cubas, 2006).

O excesso de vitamina D (hipervitaminose D) causa hipercalcemia, resultante de uma excessiva absorção intestinal do elemento, podendo levar a calcificações metastáticas em tecidos moles, especialmente nos sistemas renal, vascular (camada média de artérias elásticas como a aorta) e aparelho respiratório, além de anemia consequente à interferência do excesso da vitamina na eritropoiese (Macwhirter, 1994; Leeson e Summers, 2001; Carciofi e Oliveira, 2007). Evidências clínicas sugerem que araras jovens podem ser particularmente sensíveis a hipervitaminose $D$ com provável nefrocalcinose associada a esta afeç̧ão, tendo ainda relatos em um pombo, um tucano, um cardeal e uma variedade de psitacídeos (Macwhirter, 1994).

O ergocalciferol (vitamina D2) tem baixa atividade nas aves. Por outro lado o colecalciferol ou vitamina D3 é bastante ativo, sendo produzido por radiação ultravioleta na pele a partir do 7-deidrocolesterol, após sua absorção (intestinal ou pela pele) este é transportado via corrente sanguínea para o organismo, e ao contrário dos peixes, as aves pouco estocam esta vitamina no fígado (Leeson e Summers, 2001).

A exposição a diferentes níveis de luz UVB (ultravioleta $B$ ) também pode levar a distúrbios esqueléticos, tal como discondroplasia tibial (Dacke, 2000). Por esse motivo, é importante que aves de cativeiro não sejam mantidas em ambientes fechados, assim como se deve também atentar para o fato de que a luz solar filtrada por vidros não permite uma adequada síntese de vitamina $D$ pela pele. Esses cuidados ajudam a prevenir muitas expressões de hipocalcemia, vistas especificamente em Papagaios Cinzentos Africanos (Psittacus erithacus) e em outras espécies suscetíveis (Stanford, 2006).

Para espécies herbívoras e onívoras, a necessidade de vitamina D é suprida por síntese cutânea quando os animais são suficientemente expostos à radiação solar. Entretanto, algumas espécies de aves não apresentam essa 
biossíntese suficientemente ativa, necessitando de vitamina D3 via dieta (Carciofi e Oliveira, 2007).

\section{Cálcio e fósforo}

O excesso de fósforo presente em vísceras e algumas sementes, associado ao excesso de gordura de alguns grãos, como girassol e amendoim, pioram os desequilíbrios nutricionais destes alimentos (Carciofi e Oliveira, 2007). Por outro lado, o fósforo disponível das fontes de origem vegetal não é suficiente para atender às exigências nutricionais para 0 adequado desempenho e mineralização óssea, por isso, é necessária a suplementação com fontes inorgânicas (Rostagno et al., 2000). Normalmente o fosfato bicálcio é a principal fonte suplementar de fósforo para as aves, entretanto seu custo é elevado (Araújo, 2009).

O excesso de cálcio na dieta, por sua vez, ocasiona distúrbio nutricional grave, que acarreta hipercalcitoninismo, alteração na ossificação endocondral e menor remodelamento ósseo, além de poder agir como antagonista, dificultando a absorção de outros minerais, tais como ferro, cobre, zinco, magnésio, sódio, potássio, entre outros (Smith e Kabaja, 1984; Waldroup, 1996).

A deficiência de cálcio tem sido uma das causas de doenças nutricionais mais frequentes em animais silvestres de cativeiro (Robbins, 1983), as quais variam de osteodistrofia em jovens (em parte devido à maior exigência de cálcio em aves em fase de crescimento), a retenção de ovos em adultos e até convulsões por hipocalcemia (Rosskopf, 1985; Hochleithner, 1989; Hochleithner 1997; Harcourt-Brown, 2003). Sementes, frutas, carnes, castanhas, vísceras e insetos dificilmente apresentam mais do que 0,1\% de cálcio na matéria seca, uma quantidade irrisória, e via de regra apresentam mais fósforo do que cálcio, motivo pelo qual devem sempre ser suplementados (Fowler, 1986). Como fontes de cálcio incluem-se legumes, forragens, folhas escuras, presas inteiras, calcário, fosfato bicálcico, farinha de ossos, farinha de carne e ossos, leite e derivados lácteos (Carciofi e Oliveira, 2007). Os sintomas 
clínicos da deficiência de cálcio variam de acordo com a espécie e incluem crescimento retardado, diminuição da ingestão de alimentos, aumento da taxa metabólica basal, redução da atividade física, alterações posturais, claudicação, sensibilidade dolorosa em membros, paralisias transitórias, tetania, deformidades ósseas, tais como membros varus ou valgus, e aumento das concentrações de fosfatase alcalina sérica (Fowler, 1986). As fêmeas em postura podem apresentar ovos com casca fina ou mole, atonia de útero, distocia, retenção de ovos no oviduto e ossatura enfraquecida (Godoy e Cubas, 2006).

Misturas de sementes são tradicionalmente utilizadas para a alimentação de papagaios em cativeiro, mas possuem baixos níveis de vitamina D3 e cálcio (Rosskopf, 1985; Harcourt-Brown, 2003; Stanford, 2003a, Stanford, 2003b). Além do mais, misturas de sementes podem conter altos níveis de fósforo, que podem se ligar ao cálcio em complexos de fitato (Klasing, 1998). Amendoim e sementes de girassol são particularmente prejudiciais para a homeostase do cálcio, não apenas por conterem somente $20 \%$ da exigência diária de cálcio de um pássaro que não está em período de postura, mas também por conter alto teor de gordura que pode se combinar com o cálcio para formar sabões insolúveis no trato gastrointestinal (Petrack, 1982; Stunkard, 1984; Carciofi e Oliveira, 2007). De acordo com Godoy e Cubas (2006), a relação Ca:P é de aproximadamente $1: 7$ na semente de girassol, $1: 26$ no milho e 1:6 no amendoim.

A hipocalcemia é uma síndrome comum em aves, sendo que Papagaios Cinzentos Africanos (Psittacus erithacus) criados em cativeiro são particularmente suscetíveis a esta, embora a etiologia ainda não seja confirmada (Rosskopf, 1985; Hochleithner, 1989; Hochleithner 1997; Harcourt-Brown, 2003).

Um estudo realizado por Stanford (2003a) mostrou que é possível produzir mudanças nos parâmetros de cálcio, variando as condições de criação dos Papagaios Cinzentos Africanos. O estudo demonstrou os efeitos ao se alimentar as aves com uma dieta formulada com altos níveis de vitamina D3 e 
cálcio, em comparação com um grupo-controle alimentado com sementes. A dieta formulada produziu um aumento significativo nos níveis de cálcio ionizado e de 25-hidroxicolecalciferol em relação ao grupo alimentado com sementes (Tian et al., 1994; Stanford, 2003b).

Uma questão nem sempre fácil de resolver é de que maneira veicular o cálcio na dieta. Geralmente a quantidade de fosfato tricálcio necessária representa de 0,5 a $1,5 \%$ em dietas com elevada umidade (frutas, carnes, alimentos cozidos) e de 2 a $3 \%$ em dietas secas (misturas de grãos, por exemplo). Boas rações são uma excelente alternativa nutricional e não precisam ser suplementadas, atentando-se aos teores de cálcio, que devem ser entre 1 e 1,5\% (Carciofi e Oliveira, 2007).

\section{A contribuição do esqueleto para a formação de cascas dos ovos}

Em animais adultos, deve-se atentar para os níveis de cálcio relacionado à formação da casca do ovo devido à mesma ser formada por carbonato de cálcio. Em galinhas, durante as cinco primeiras horas de formação de casca a deposição de carbonato de cálcio aumenta rapidamente até atingir $300 \mathrm{mg} /$ hora e no final do período cerca de $800 \mathrm{mg}$ deste elemento estará na casca (Leeson e Summers, 2001).

O esqueleto da maioria dos pequenos passeriformes contém de três a cinco vezes a quantidade de cálcio presente em um único ovo (Graveland e Gijzen, 1994). A medula óssea dessas aves é um tipo de osso lábil a partir do qual o cálcio pode ser mobilizado mais rapidamente do que do osso estrutural (Simkiss, 1967; Hurwitz, 1987).

Aves em uma dieta deficiente de cálcio mantém a quantidade de osso medular constante, por meio da conversão do osso estrutural (Graveland e Gijzen, 1994). O cálcio na medula, portanto, provavelmente não serve como uma reserva de cálcio por si só, mas atua como um amortecedor contra os picos da demanda de cálcio durante o curto período de formação da casca (Ankney e Scott, 1980; Etches, 1987; Hurwitz 1987). 
Há duas maneiras utilizadas pelas aves para a captação de nutrientes na época de postura. Na primeira, as aves acumulam esses nutrientes por um longo período, antes da reprodução; na segunda, as aves ingerem esses nutrientes pouco tempo antes da postura (Drent e Daan, 1980; Thomas, 1988; Winkler e Allen, 1996).

\section{Osteodistrofias}

De acordo com Pond et al. (1995), as osteodistrofias podem ocorrer por alterações na ingestão de cálcio, fósforo, vitamina D, proteína, cobre, zinco, manganês e vitamina $A$. Os nutrientes mais frequentemente envolvidos nas osteodistrofias são: o cálcio, o fósforo e a vitamina D.

As osteodistrofias podem ser divididas em osteopenias e osteomegalias, segundo uma classificação morfopatogênica. As osteopenias incluem osteoporose, raquitismo, osteomalácia (decorrente da falta de fósforo ou da deficiência de vitamina $D$ em adultos) e osteodistrofia fibrosa (hiperparatireoidismo nutricional secundário). As osteomegalias decorrem de processos multifatoriais, podendo ter etiologia genética, nutricional ou ambiental (Carciofi e Oliveira, 2007).

A condrodistrofia ou desvio angular das patas é uma afecção resultante da redução da capacidade de crescimento da cartilagem epifiseal, associada a um desarranjo da formação colunar das células e a uma maior proliferação da matriz da cartilagem. Essa perda do arranjo colunar afeta a vascularização da cartilagem, resultando em diminuição do crescimento longitudinal do osso que, apesar desse fato, continua crescendo em diâmetro (Riddell, 1981); ou seja, ocorre malformação da articulação tibiotarsal, encurtamento e espessamento da tíbia. Nos casos graves, o tendão gastrocnêmio desvia-se para fora dos côndilos da articulação tibiotarsal (Julian, 2005; Carciofi e Oliveira, 2007). As formas valgus (desvio lateral) e varus (desvio medial) ocorrem (Schmidt et al., 2003), sendo a forma varus a mais comum (Carciofi e Oliveira, 2007); porém, em um estudo realizado por Bernardi (2011) em frangos de corte, a forma valgus foi a mais comum. Apesar de a etiologia desta osteomegalia ainda não 
ser bem esclarecida, sabe-se que a deficiência de alguns nutrientes como manganês, cobre, colina, zinco e vitaminas do complexo B (Schmidt et al., 2003), assim como o excesso de cálcio, fósforo ou desbalanços na relação desses dois nutrientes, podem causá-la. Entretanto, mesmo aves que recebem dietas com perfil nutricional adequado podem ser acometidas. A condrodistrofia também acomete filhotes aos quais são oferecidas dietas de alta energia e ou alta proteína e que ficam restritos a recintos pequenos que limitem suas possibilidades de exercício, o que resulta em crescimento rápido e sobrepeso, levando a distúrbios de desenvolvimento osteoarticular. Essa enfermidade afeta diversas espécies de aves em crescimento, tais como gansos, cisnes, perus, galinhas, patos, ratitas, entre outras (Carciofi e Oliveira, 2007); porém não é comum ocorrer em aves de companhia como os psitacídeos e passeriformes (Schmidt et al., 2003).

O hiperparatireoidismo é uma desordem metabólica expressa clinicamente por distúrbios na mineralização e homeostase ósseas, devido à secreção excessiva de PTH. O hiperparatireoidismo é classificado em primário e secundário, sendo o secundário de origem renal ou nutricional. Nos animais domésticos a incidência de hiperparatireoidismo secundário é a mais comum. Nesses casos, a glândula paratireoide reage a um distúrbio patológico que tenha afetado a homeostase do cálcio (Stanford, 2005). Sintomas específicos das aves com hiperparatireoidismo nutricional secundário incluem: retenção de ovo, prolapso cloacal, fraturas patológicas, deformidades angulares dos ossos, diminuição da qualidade da casca do ovo, afundamento do externo, deformações de coluna, estreitamento de pelve e problemas locomotores (Bauck, 1995; Kollias, 1995).

A osteodistrofia fibrosa surge como uma evolução do hiperparatireoidismo nutricional secundário, quando este não é corrigido, devido à estimulação crônica do PTH sobre os ossos (Woodward, 1996; Schmidt et al., 2003). O tecido fibroso e tecido ósseo reativo possuem menos integridade estrutural, predispondo os animais a deformações e a fraturas patológicas (Taylor e Dacke, 1984). De acordo com Carciofi e Oliveira (2007), 
a osteodistrofia fibrosa pode ser hipo-osteótica (menos osso), iso-osteótica ou hiper-osteótica (mais osso, porém de pior qualidade). Verifica-se em filhotes, mais frequentemente, a osteodistrofia hipo-osteótica, caracterizada por osteopenia à radiografia, entortamento dos ossos longos e fraturas em galho verde. Em adultos, pode ser observada a osteodistrofia hiper-osteótica.

Para espécies que aceitam folhas verdes, estas podem representar uma fonte de cálcio, mas a suplementação via ração adequada ou o emprego de compostos minerais é sempre recomendável, visto que o hiperparatireoidismo nutricional é tipicamente observado em animais alimentados com dieta pobre em cálcio, mas com altos níveis de fosfato, ou dietas com inadequada concentração de vitamina D3 (Stanford, 2005).

A Osteoporose é uma osteopenia caracterizada por uma redução na massa óssea (falha na formação da matriz óssea), com o osso remanescente normalmente mineralizado. Na osteoporose existe perda de colágeno e apatita, alterações nas propriedades físicas das fibras de colágeno; além disso, há aumento da hidroxilação de lisina e mudanças no perfil crosslink intermolecular que levam ao aumento de colágeno e consequentemente aumento da fragilidade óssea. A espessura do osso cortical fica reduzida e este se torna mais poroso; o osso trabecular se torna mais fino e, eventualmente, é perdido. Ossos nestas condições podem facilmente sofrer fraturas e podem se dobrar caso uma pressão for aplicada. As causas da osteoporose incluem deficiência de cálcio, cobre, fósforo ou de vitamina D3, atividade física reduzida e anorexia (Schmidt et al., 2003).

O raquitismo ocorre em aves jovens e refere-se à mineralização insuficiente da matriz óssea, decorrente da deficiência de cálcio, fósforo e/ou vitamina $\mathrm{D}$ na dieta ou pela exposição insuficiente à luz ultravioleta (Riddell, 1981; Godoy e Cubas, 2006).

O tratamento das osteopenias tem como fator principal a correção da dieta, sendo que $o$ raquitismo é tratado com vitamina $D$ e $o$ hiperparatireoidismo nutricional secundário, com cálcio. Tendo o cálcio e o fósforo como macroelementos, as dietas podem ser suplementadas com 
fosfato tricálcio ou com suplemento vitamínico-mineral em pó, sendo que este possui a vantagem de suplementar outros minerais e vitaminas (Carciofi e Oliveira, 2007), tendo em vista que os psitaciformes são incapazes de sintetizar a maioria das vitaminas que necessitam (Stanford, 2005). A quantidade a ser suplementada dependerá da quantidade de cálcio na dieta e da necessidade do elemento pela espécie. Apesar de existirem suplementos líquidos de cálcio, fósforo e vitamina $D$, esses são menos efetivos, pois apresentam muita vitamina $D$ e pouco cálcio, que é um elemento essencialmente insolúvel, tornando a quantidade necessária para suplementar o cálcio impraticável (Carciofi e Oliveira, 2007). Além disso, deve-se dar preferência ao emprego de vitamina D3 à vitamina D2, pois a ligação da vitamina D2 com as proteínas plasmáticas nas aves é menos eficiente do que em mamíferos (Deluca et al., 1988).

O tratamento das osteodistrofias é longo, podendo demorar meses até a remineralização óssea $e$, às vezes, pode incluir cirurgias ortopédicas, imobilização e o uso de anti-inflamatórios. Contudo, a maior parte das alterações anatômicas do tecido ósseo é irreversível e o animal estará permanentemente comprometido como, por exemplo, no caso de aves que manifestam osteoporose e não respondem à correção ou suplementação da dieta (Mazzuco, 2007). Porém, filhotes ainda muito novos, tratados no início da doença, são passíveis de recuperação (Carciofi e Oliveira, 2007).

Para tratamento emergencial de aves hipocalcêmicas, segundo Godoy e Cubas (2006), o método utilizado é a aplicação de cálcio (gluconato de cálcio ou outros) por via endovenosa, intramuscular ou intraóssea.

\section{Considerações Finais}

O cálcio e o fósforo são elementos essenciais na constituição do organismo de qualquer espécie animal e nas aves não é diferente, sendo essenciais desde as funções metabólicas até para a formação da casca do ovo. Problemas como retenção de ovo e diminuição da qualidade da casca influenciam diretamente na reprodução e na perpetuação das espécies. 
BAGGIO JÚNIOR, R. e PITA, M.C.G. A importância do cálcio e fósforo na nutrição de psitacídeos e passeriformes - uma revisão. PUBVET, Londrina, V. 7, N. 19, Ed. 242, Art. 1596, Outubro, 2013.

Nesse contexto, o trabalho visou a mostrar a importância de as aves terem uma nutrição adequada. No Brasil há a cultura de que a alimentação das aves somente com grãos é suficiente para uma boa nutrição; porém, pode-se notar que mediante essa dieta os animais ficam obesos e desnutridos, sabendo-se que os grãos são, principalmente, fonte de lipídeos.

Portanto, aves criadas em cativeiro (criações comerciais e zoológicos, entre outros) devem receber uma nutrição balanceada, sendo uma boa alternativa a alimentação com rações. Atualmente existem rações específicas para passeriformes, psitacídeos e outros, nas quais os teores de minerais, proteínas, carboidratos e gordura são adequados a cada espécie, sendo desnecessária a suplementação.

\section{Referências Bibliográficas}

ANKNEY, E.D. \& D.M. SCOTT. Changes in nutrient reserves and diet of breeding Brownheaded Cowbirds. p.684-696, 1980.

ARAÚJO, L. F. Uso de fosfatos com diferentes relações flúor:fósforo na alimentação de poedeiras semipesadas na fase de produção. Revista Brasileira de Zootecnia, v.38, n.6, p.1033-1036, 2009.

ASLAM S.M., GARLICH J.D., QURESHI M.A. Vitamin D deficiency alters the immune responses of broiler chicks. Poultry Science v.77, n.6, p.842-849, 1998.

ASSOCIATION OF AMERICAN FEED CONTROL OFFICIALS INCORPORATED. Nutrition expert panel review: new rules for feeding pet birds. Official Publication - Feed Management, Atlanta, v.49, n.2, 1998.

BAUCK, L. Nutritional problems in pet bird. Sem. Avian Exotic Pet Med., v.4, n.1, p.3-8, 1995.

BENTLEY, P.J. Comparative Vertebrate Endocrinology. Cambridge, UK, Cambridge Univ. Press, p.269-301, 1998.

BERNARDI, RODRIGO. Problemas locomotores em frangos de corte. Dissertação de mestrado. Dourado, MS: UFGD, 2011.

BUCKLEY, J.C. Pathophysiologic considerations of osteopenia. Compend Contin Educ Pract Vet v.6, p.552-560, 1984.

BUSH, B.M. Interpretation of laboratory results for small animal clinicians. 1 ed., London: Blackwell Scientific Publications, p.350-386, 1991.

CARCIOFI, A. C; OliveIRA, L. D. Doenças Nutricionais. Em: CUBAS, S. C.; SILVA, J. C. R.; CATÃO-DIAS, J. L. Tratado de Animais Selvagens. São Paulo: Ed. Roca, p.838-847, 2007.

DACKE, G.C. Em GC Whittow (ed): Sturkie's Avian Physiology 5th ed. London, UK, Academic Press, p.472-485, 2000. 
DELUCA, H.F.; NAKADA, M.; TANAKA, Y.; SCINSKI, R.; PHELP, M. The plasma binding protein for vitamin $D$ is a site of discrimination against vitamin $D-2$ in the chick. Archives of Biochemistry and Biophysics v.965, p.16-21, 1988.

DRENT, R.H; DANN, S. The prudent parent: Energetic adjustments in avian breeding. Ardea 68, p.225-252, 1980.

DUNCAN, R.J.; PRASSE, K.W. Veterinary laboratory medicine clinical pathology 2 ed. Ames: Iowa State University, p.181-200, 1986.

EARLE KAY, E.; CLARKE NIGEL, R. Nutrition of Caged Birds: The Nutrition of the Budgerigar (Melopsittacus undulatus). The Journal of Nutrition, p.186-192, 1991.

ETCHES, R.J. Calcium logistics in the laying hen. J. Nutr. v.117, p.619-628, 1987.

FOWLER, M.E. Metabolic bone disease. Em: FOWLER, M.E. Zoo and Wild Animal Medicine. 2 ed. Philadelphia: Saunders. p.69-90, 1986.

GODOY, S.N.; CUBAS, Z.S. Algumas doenças de aves ornamentais, 2006. Disponível em http://www.canarilalmada.com/ download/download/Dossierdedoencas.pdf Acesso em: 10/01/2013.

HARCOURT-BROWN, N.H. Incidence of juvenile osteodystrophy in handreared grey parrots (Psittacus erithacus). Vet Rec 152, p.438-439, 2003.

HOCHLEITHNER, M. Convulsions in African grey parrots in connection with hypocalcemia: Five selected cases. Proc 2nd Euro Symp Avian Med Surg, p.44-52, 1989.

HOCHLEITHNER, M.; HOCHLEITHNER, C.; HARRISON, G.J. Evidence of hypoparathyroidism in hypocalcemic African grey parrots. The Avian Examiner Special Suppl. HBD International Ltd, Spring, 1997.

HURWITZ, S. Effect of nutrition on egg quality. Em: R.G. Wells \& e.G. Belyavin (eds). Egg Quality - Current Problems and Recent Advances. Poultry Science Symposium 20, Butterworths, London, p.235-254, 1987.

GRAVELAND, J.; GIJZEN, T. V. Arthropods and seeds are not sufficient as calcium sources for shell formation and skeletal growth in passarines. Netherlands Institute of Ecology, Centre for Terrestrial Ecology, Heteren, p.299-314, 1994.

JULIAN, R. Patologias ósseas em aves. Em: Conferência APINCO 2005 de ciência e tecnologia avícolas, Campinas. Volume 2, Campinas: FACTA, p.107-122, 2005.

KLASING, K.C. Comparative Avian Nutrition. New York, CAB Intl, p.290-295, 1998.

$\mathrm{KOCH}, \mathrm{J}$; ; et al. Blood ionic calcium response to hypocalcemia in the chicken induced by ethylene glycol-bis-(B-aminoethylether) $\mathrm{N}, \mathrm{N}^{\prime}$-tetra-acetic acid: Role of the parathyroids. Poult Sci 63, p.167-171, 1984.

KOLLIAS, G. V. Diets, feeding practices, and nutritional problems in psittacine birds. Vet. Med., p.29-39, 1995.

LEESON, S. SUMMERS, J. D. Nutrition of the chicken. 4th Edition, ed. University Books, Ontario Canada, p.591, 2001.

MACARI, M.; FURLAN, R. L.; GONZALES E. Fisiologia aplicada a frangos de corte. Jaboticabal, FUNEP/UNESP p.375, 2002.

MACWHIRTER, P. Avian Medicine: Principles and Application. Florida: Ed. Wingers Publishing Inc., p.842-861, 1994. 
MAZZUCO, H. Osteoporose em poedeiras comerciais: uma doença metabólica multifatorial. Embrapa suínos e 2007.2 Disponível em: http://www.boletimpecuario.com.br/notes/noticia.php?not=ancora2745. boletimpecuario.

Acesso em: 17/02/2013.

MCDONALD, D. Nutritional Considerations Section I, Nutrition and Dietary Supplementation (Chapter 4). Em: Harrison, G. J. and Lightfoot, T. L. Clinical Avian Medicine - Volume I. Spix Publishing Inc, Palm Beach, FL, USA, p.86-107, 2006.

MCDONALD, L. J. Hypocalcemic Seizures in an African Grey Parrot. Can Vet J Volume 29, p.928-930, 1988.

NELSON, W.R.; TURNWALD, G.H.; WILLARD, M.D. Endocrine, metabolic, and lipid disorders. In: WILLARD, M.D.; TVEDTEN, H.; TURWALD, G.H.; Small animal clinical diagnosis in laboratory methods. 2 ed. Philadelphia: W.B. Saunders, p.147-151, 1994.

PETRACK, M.L. Diseases of Cage and Aviary Birds. 2 ed. Philadelphia: Lea \& Febiger, p.223228 e p.485-486, 1982.

POND, W. G.; CHURCH D. C.; POND, K. R. Basic Animal Nutrition and Feeding. 4 ed., New York: John Wiley, p.615, 1995.

RIDDELL, C. Skeletal deformities in poultry. Advances in Veterinary Science and Comparative Medicine, v.25, p.277-310, 1981.

ROBBINS, C. T. Wildlife Feeding and Nutrition. 1 ed. San Diego: Academic Press Inc., p.343, 1983.

ROSSKOPF, W.J.; WOERPEL, R.W.; LANE, R.A. The hypocalcemic syndrome in African greys: An updated clinical viewpoint. Proc Assoc Avian Vet, p.129-132, 1985.

ROSTAGNO, H.S.; ALBINO, L.F.T.; DONZELE, J.L. et al. Composição de alimentos e exigências nutricionais de aves e suinos. Tabelas brasileiras para aves e suinos. Viçosa, MG: Editora UFV, p.141, 2000.

RUNHO, R. C.; GOMES, P. C.; ROSTAGNO, H.S.; ALBINO, L. F. T.; LOPES, P. S.; POZZA, P. C. Exigências de fósforo disponível para frangos de corte machos e fêmeas de 1 a 21 dias de idade. Revista Brasileira de Zootecnia, v.30, n.1, p.187-196, 2001.

SCHMIDT, R. E.; REAVILL, D. R.; PHALEN, D. N. Pathology of pet and aviary birds. Iowa: Ed. Blackwell, p.149-163, 2003.

SENA, S.F.; BOWERS, G.N.J. Measurement of ionized calcium in biological fluids: ion-selective electrode method. Meth. Enzimol., v.158, p.320- 334, 1988.

SIMKISS, K. Calcium in reproductive physiology. Chapman \& Hall, 1967.

SMITH, O. B.; KABAJA, E. Effect of high dietary calcium and wide calcium/phosphorus rations in broiler diets. Poultry Science, v.64, p.1713-1720, 1984.

STANFORD M.D. Measurement of ionised calcium in grey parrots: The effect of diet. Proc Euro Assoc Avian Vet, 2003a.

STANFORD M.D. Measurement of 25-hydroxycholecalciferol in grey parrots: The effect of diet. Proc Euro Assoc Avian Vet, 2003b.

STANFORD M. D. Calcium metabolism in grey parrots: the effects of husbandry. 2005.

STANFORD, M. D. Calcium Metabolism (Chapter 5) Em: Harrison, G. J. and Lightfoot, T. L. Clinical Avian Medicine - Volume I. Spix Publishing Inc, Palm Beach, FL, USA, p.141-151, 2006.

STUNKARD, J.A. Diagnosis, Treatment and Husbandry of Pet Birds. 2 ed. Stunkard Publishing Co., 1984. 
TAYLOR, T.G.; DACKE, C.G. Calcium metabolism and its regulation. Em: Freeman BM (ed) Physiology and Biochemistry of the Domestic Fowl Volume 5. London, UK, Academic Press, p.125-170, 1984.

THOMAS, V. G. Body condition, ovarian hierarchies, and their relation to egg formation in anseriform and galliform species. Acta XIX Intern. Ornith. Congress I, p.353-363, 1988.

TIAN, X.Q.; CHEN, T. C.; LU, Z; SHAO, Q; HOLICK, M. F. Characterization of the translocation process of vitamin D3 from the skin into the circulation. Endocrinology v.135, n.2, p.655-6121, 1994.

UNDERWOOD, E. J.; SUTTLE, N. F. Mineral nutrition of livestock. 3 ed. London: CAB international, p.614, 1999.

WALDROUP, P.W. Bioassays remain necessary to estimate phosphorus, calcium bioavailability. Feedstuffs, v.68, p.13-20, 1996.

WALSH, M.T. Seizuring in pet birds. Proc Annu Meet Assoc Avian Vet p.121-128, 1985.

WINKLER, D. W. \& ALLEN, P. E. The seasonal decline in tree swallow clutch size: physiological constraint or strategic adjustment? Ecology v.77, p.922-932, 1996.

WOODWARD, J. Metabolic bone disease. Em: Veterinary Pathology Philadelphia, U.S.A., p.915923, 1996. 